\title{
Level of medication self-management capacity among patients on ambulatory care exiting hospital pharmacy at Muhimbili National Hospital, Tanzania: a descriptive cross-sectional study
}

Hamu Mlyuka ${ }^{1 *}$, Hija Salehe ${ }^{1}$, Wigilya Mikomangwa ${ }^{1}$, Manase Kilonzi ${ }^{1}$, Alphonce Marealle ${ }^{1}$, Ritah Mutagonda ${ }^{1}$ and George M. Bwire ${ }^{2}$

\begin{abstract}
Objectives: Medication management capacity of a patient on ambulatory care is direct related to adherence. To our knowledge data on medication management capacity for ambulatory care patients exiting outpatient pharmacy outlets in Tanzania are scarce. This study aimed to determine the level of medication management capacity among patients on ambulatory care exiting Muhimbili National Hospital outpatient pharmacy outlet.

Results: A total of 424 patients on ambulatory care participated in the study. Three hundred eighty-seven (91.3\%) out of 424 interview questionnaires had complete data and qualified for data analysis. Majority (62.3\%) out of 387 study participants had poor medication management capacity; $65.3 \%$ out of 387 patients were unable to correctly read the prescription and match the drugs they are carrying. More than half (57.4\%) out of 387 participants were unable to correctly take the dose, $73.9 \%$ out of 387 were unable to correctly tell the dosing frequency and duration. Only $10.6 \%$ out 155 patients with prescription containing drugs with warning or precaution or contraindication or potential side effects were aware.
\end{abstract}

Keywords: Medication self-management, Medication management capacity, Ambulatory care

\section{Introduction}

Adherence to medication is a key determinant of regimen effectiveness and safety. It is also a mediator of medical practices and patients' outcomes [1]. Almost $20-50 \%$ of patients do not take their medications as prescribed and the situation is even worse in chronic condition [2]. In USA non-adherence causes 125,000 death and $10 \%$ hospital admission annually. Enormous healthcare financial burden up to US $\$ 289$ billion per anum are attributed by medication non adherence [3].

\footnotetext{
*Correspondence: hmlyuka2011@gmail.com

${ }^{1}$ Clinical Pharmacy and Pharmacology Department, School of Pharmacy, Muhimbili University of Health and Allied Sciences, P.O. Box 65013, Dar es Salaam, Tanzania

Full list of author information is available at the end of the article
}

Adherence to medication is direct related to medication management capacity (MMC) of an individual patient $[4,5]$. MMC is defined as "the cognitive and functional ability to self-administer a medication regimen as it has been prescribed" [6]. Functional skills like correctly identifying the medication, opening the container, taking the right dose and timing the use of medication are components of medication self management. In an emphasis of this relationship Bailey and colleagues developed "medication self-management model" which aims to deconstruct the tasks involved in taking medication [7]. Medication management capacity tell us the accuracy of using medication while adherence based on pill count, refill or self-report provide an insight of how often one is taking the medication. The intervention focusing 
on boosting patients $\mathrm{MMC}$ have resulted into reduction in emergency department visiting and hospital admission [8].

MMC can be measured using Drug Regimen Unassisted Grading Scale (DRUGS) or Medication Management Ability Assessment (MMAA) [6, 9]. DRUGS is used to measure MMC by considering the four steps which are identification of the right medication, accessing by opening the container, picking the correct number/amount of tablet or syrup and providing elaborative timing of using the medication [6].

So far, in Tanzania the data on level of medication management are dearth. This study aimed to assess the level of medication management capacity among clients exiting hospital pharmacy outlets at Muhimbili National Hospital, Dar es Salaam Tanzania.

\section{Main text \\ Methods \\ Study area and design}

This was a descriptive cross-sectional study conducted from February to July, 2018 at Muhimbili National Hospital (MNH) outpatient pharmacy outlets. $\mathrm{MNH}$ is a national referral tertiary level research center and university teaching hospital with 1500 beds facility, attending 1000 to 1200 outpatients per day. The hospital is well equipped with laboratories, modern diagnostic facilities, specialized clinics as well as highly skilled manpower in all cadres of healthcare providers.

\section{Study population and sample size}

To be included in this study participant was supposed to be; (1) from outpatient clinic in MNH (2) carrying a prescription with more than one drug for oral use (3) carrying medicines he/she has received from pharmaceutical personnel at MNH outpatient pharmacy outlets (4) not blind (5) not deaf (6) not drunk (7) not a guardian (8) with a regimen of more than three days of use. Owing to scarcity of data on prevalence of level of medication management capacity (MMC) the $50 \%$ proportion was set as a reference population proportion to calculate the sample size. At the Z-score of 1.96 and margin of error of $5 \%$ the sample size was 385 . To consider for partial filled or inconsistency $10 \%$ of initial sample was added to make 424.

\section{Sampling technique}

Consecutive sampling was employed to recruit study participants. The researcher stayed at the exit point of the outpatient pharmacy building. When the client was about to get outside the pharmacy building the researcher requested him/her for a brief discussion. If the client agreed, the purpose of the study was explained, and then he/she signed freely obtained consent form. Assessment for inclusion and exclusion criteria was done and those who fulfilled requirements were asked to continue with the interview.

\section{Data collection}

Face-to-face interview using semi-structured questionnaire was used to gather data for fulfilling this study objectives. The questionnaire consisted questions and directives to assess medication self management capacity. This was a minor modified adoption of DRUGS tool which was used by Kripalani and colleague [6] in assessing MMC. On medication identification part; patient was asked to read what has been written on the prescription, show the particular drug, tell about warning, precaution or contraindication, important possible side effects and interactions with food or other drugs on the prescription. The researcher used Medscape interaction checker online software to check for potential life threatening interaction and match with the participant's response. The patient was then asked to open the identified drug and the researcher observed the process of opening. After opening the container, the patient was asked to tell the number of tablet(s) or amount of syrup/suspension/ solution which he/she is suppose to be taking per dose. Finally the patient was asked to tell the researcher about intervals of time to take the next dose and the total duration he/she will be using the medication. The steps were repeated for each of the medication on the prescription. For each question, the overall response was correct only if the participant has managed to perform a particular task correctly for all the prescribed medications on the prescription. Modified Bloom cut off point from study by Abdullah and colleagues [10] was used to categorize the MMC of the participants. The MMC was rated as poor/ inadequate (score $<50 \%$ ), moderate (score: $50-59 \%$ ), good (score: $60-69 \%)$, very good (70-79\%) and excellent (80-100\%).

\section{Data analysis}

Analysis was done using statistical package for social sciences (SPSS) version 23. Categorical data like gender, age groups, levels of education, level of MMC were summarized using frequency distribution and bar charts.

\section{Results}

A total of 424 patients on ambulatory care participated in this study. Three hundred eighty-seven (91.3\%) questionnaires out of 424 were properly filled and qualified for data analysis. Patient characteristics are summarized on Table 1. Majority of the study participants $(66.4 \%)$ were women. Many participants aged between 30 and 49 years and more than half attained secondary level of education. 
Table 1 Patients socio-demographic characteristics

\begin{tabular}{lc}
\hline Characteristics $\mathbf{N}=\mathbf{3 8 7})$ & $\mathbf{n}(\%)$ \\
\hline Gender & \\
Male & $130(33.6)$ \\
Female & $257(66.4)$ \\
Age, years & \\
$20-29$ & $32(8.30)$ \\
$30-39$ & $133(34.4)$ \\
$40-49$ & $145(37.5)$ \\
$50-59$ & $50(12.9)$ \\
$>60$ & $27(7)$ \\
Marital status & \\
Single & $66(17.1)$ \\
Married & $218(56.3)$ \\
Divorced & $21(5.4)$ \\
Widowed & $19(4.9)$ \\
Cohabit & $63(16.3)$ \\
Education level & \\
Never & $15(3.9)$ \\
Primary & $88(22.7)$ \\
Secondary & $208(53.7)$ \\
Tertiary & $76(19.6)$ \\
\hline
\end{tabular}

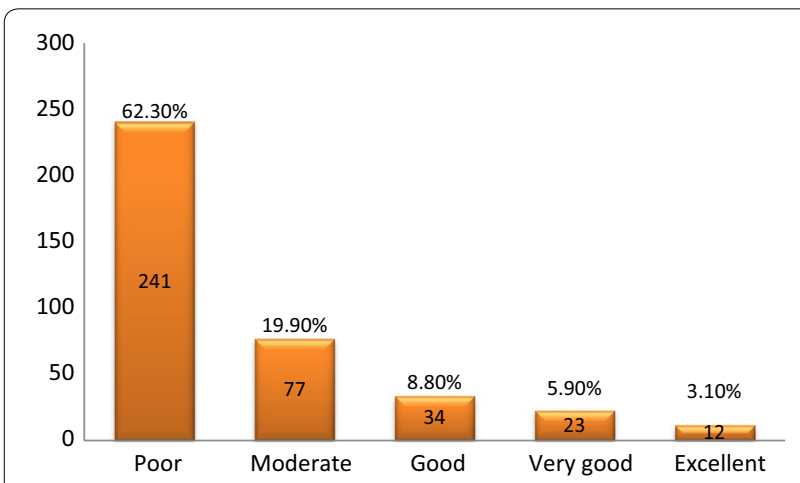

Fig. 1 Bar chart of prevalence of medication management capacity

\section{Patients' medication management capacity}

The overall grading showed that 241 (62.3\%) of 387 study participants had poor MMC (Fig. 1). Majority of these participants were not able to identify, take the right dose or timing the frequency and duration as detailed below.

Table 2 shows the response to questions and activities to measure MMC. More than half of study participants (65.3\%) were not able to correctly read and interpret the instructions written on their prescriptions and match with the medications on hand. All patients demonstrated an ability to open a container and take the medication. When asked to count pills or measure the mockup amount as directed on the prescription $222(57.4 \%)$ were
Table 2 Response to attributes of measuring medication self management

Patient's responses on medication self management questions or activities $(\mathrm{N}=387)$

$$
\begin{aligned}
& \text { Item question or activity } \\
& \text { Proportion of response (\%) }
\end{aligned}
$$
Correct
42.6

$\begin{array}{ll}\text { Able } & 34.7 \\ \text { Unable } & 65.3\end{array}$

$\begin{array}{ll}\text { Correct } & 26.1 \\ \text { Incorrect } & 73.9\end{array}$

6. What is a possible potential side effect/warning/precaution due to your medication? ${ }^{\mathrm{a}}$ (aware only if explained correctly for all drugs with possible side effects/warning/precaution) $n=155$

$\begin{array}{ll}\text { Aware } & 10.6 \\ \text { Unaware } & 89.4\end{array}$

7. What is your key source of medication information?

Medical personnel $\quad 40$

Internet 53

Others (bulletin, brochures, banners, 7 etc.)

8. Are you aware of drug interaction?

$\begin{array}{ll}\text { Aware } & 76 \\ \text { Unaware } & 24\end{array}$

9. Can you tell the possible dangerous interaction with regards to your

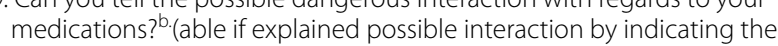
drugs on the $R x) n=60$

$\begin{array}{ll}\text { Able } & 7 \\ \text { Unable } & 93\end{array}$

a The total number of prescription with potential side effects/warning/ contraindication/precaution was 155 hence denominator for the proportions of response to item

b The total number of prescription with possible potential interaction was 60 hence the denominator for proportions of response to the item

not able to correctly take the prescribed dose. When asked to explain the interval of hours to take the next dose and duration for each of the prescribed medications only $26.1 \%$ out of 387 participants were able to explain correctly. 


\section{Discussion}

This study found an alarming level of prevalence of poor medication self-management among patients on ambulatory care. Many patients had difficulties on reading and interpreting what has been written on the prescription matching with what they are carrying on the hand. The medication self-management model has six tasks i.e. fill, understands, organize, take, monitor and sustain [7]. The scope of this study was to measure the ability of patients to understand the prescription, organizing the plan to take medication as prescribed, ability to open a container and take right number or amount of the medications, patient's ability to monitor for side effects or warnings or interactions and the ability to sustain taking medication by understanding timing and duration of the regimen. Understanding the regimen on the prescription, taking the correct number or amount of dose, and timing of doses and duration of the regimen are areas which troubled many participants in this study. These findings concurred with what was observed in India after Gupta and colleagues conducted a study on the impact of patient information leaflets on medication adherence. Many patients had difficulties in timing of taking medications and duration of the regimen [11]. It is possible that, poor understanding of what has been written on the prescription or narrated by the pharmaceutical personnel is the root cause of poor performance on many of the tasks as testified in the work of Wolf and colleagues [12]. Majority of study participants reported to be secondary level graduates. Therefore, tough the study didn't focus on associated factors; it is most probable that education level of the study participants had a major influence of MMC as it was reported in study by Kripalani et al. [6] and in the study which was conducted in Ethiopia [13].

With regard to medication information seeking behavior, this study has shown that more than half of the patients were not eager to ask their healthcare provider issues concerning their medications. This finding second what was observed in study which was conducted in South Africa [14].Tough it was not in the scope of this study, the reason may be uncomfortable platform of hospital pharmacy facilities, long waiting queue, unfriendly and brief contact time. All these have impact on ability of the patient to comprehend with written and verbal communication which play big role in understanding the regimen [15].

In this study many patients were not aware of the side effects or warning label or contraindication or precaution or drug interactions for their medications on hand. This is in line with findings from review by Bailey and colleagues [7] and study conducted in India [11]. This increase the risk of medication errors and adverse drug reactions which may jeopardize adherence to prescribed regimen. Most probable low level of education among study participants and brief contact time with healthcare providers may be the possible reasons [16].

Majority of the participants had poor ability to pick the correct number or amount of medications. The key reason may still be the same a poor understanding of the prescription regimen means poor understanding of the downstream tasks $[6,7]$.

Owing to detrimental consequences of poor MMC different implementation researches have come out with solution to mitigate the problem. Whenever possible, pharmaceutical personnel should use the principle of universal assumption i.e. assuming all patients have poor MMC [17]. But to maximize resources allocation identification of patient with poor MMC is the first important step. Normally these patients prefer no company, fail to establish rapport, tend to just release the written papers for a caregiver to read and take actions, use initials instead of signature and have unique ways (e.g. color, symbols) of identifying pills [6, 17].

Therefore, pharmaceutical personnel should identify poor MMC patients and exercise clear health communication skill strategies using plain language and teachback method [18]. Also, proper medications packaging, labeling and dispensing using reading materials and language or symbol understood by patient [19] have proved effective to counter limitation of MMC [20]. Furthermore, intensive counseling and close follow-up are very important to patients with any MMC limitation [21, 22]. Volunteered social support on emotional, medication information, health reminder and tangible support are very important to patients with poor MMC because of their reluctance to seek help [23]. Continual training of healthcare providers on awareness, sensitization and collaboration to address the impact of poor MMC is important [24].

\section{Conclusion}

The level of MMC for majority of outpatients attending national tertiary level hospital is very low. Further study need to focus on level of MMC and associated factors.

\section{Limitations}

Consecutive sampling posed the risk of selection bias. Confounders like English proficiency as a communication language barrier, differences in cognitive and health literacy levels of the study participants were not measured. Also, sampled participants were from tertiary level hospital, with diversity in diseases and severity plus treatment durations. 


\section{Abbreviations}

MMC: medication management capacity; DRUGS: Drug Regimen Unassisted Grading Scale; MMAA: Medication Management Ability Assessment; $\mathrm{MNH}$ : Muhimbili National Hospital; Rx: prescription.

\section{Acknowledgements}

The authors highly appreciate patients on ambulatory care exiting Muhimbil National Hospital outpatient pharmacy outlets for participating in this study.

\section{Authors' contributions}

HM designed the study, data analysis and drafted the initial manuscript. HS coordinated and monitored data collection. WM, MK, AM, RM and GB participated in study design, interpretation of data and manuscript development. All authors read and approved the final manuscript.

\section{Funding}

Not applicable.

\section{Availability of data and materials}

The dataset generated and/or analyzed during this study are available from the corresponding author upon reasonable request.

\section{Ethics approval and consent to participate}

The study was reviewed and approved by Muhimbili University of health and allied sciences research ethics committee and permission to conduct research was obtained from MNH training and research directorate. Researcher explained the purpose of the study to the patients. Patients willing to participate in the study were asked to sign freely obtained written consent form prior to commencement of interview. To ensure confidentiality, only numbers were used to identify participants and the questionnaires were kept under custodian of researchers only.

\section{Consent for publication}

Not applicable.

\section{Competing interests}

The authors declare that they have no competing interests.

\section{Author details}

${ }^{1}$ Clinical Pharmacy and Pharmacology Department, School of Pharmacy, Muhimbili University of Health and Allied Sciences, P.O. Box 65013, Dar es Salaam, Tanzania. ${ }^{2}$ Department of Pharmaceutical Microbiology, Muhimbili University of Health and Allied Sciences, P.O. Box 65013, Dar es Salaam, Tanzania.

Received: 13 August 2019 Accepted: 30 October 2019 Published online: 07 November 2019

\section{References}

1. Krousel-Wood M, Muntner P, Islam T, Morisky D, Webber L. Barriers to and determinants of medication adherence in hypertension management: perspective of the cohort study of medication adherence among older adults (CoSMO). Med Clin North Am. 2009;93(Suppl 3):753-69.

2. Kripalani S, Yao X, Haynes B. Interventions to enhance medication adherence in chronic medical conditions: a systematic review. Arch Intern Med. 2007; 167:540-50.

3. Viswanathan M, Golin C, Jones C, Ashok M, Blalock S, Wines R, CokerSchwimmer E, Rosen D, Sista P, Lohr K. Interventions to improve adherence to self-administered medications for chronic diseases in the united states: a systematic review. Ann Intern Med. 2012;157:785-95.

4. Janson S, McGrath W, Covington J, Cheng S, Boushey H. Individualized asthma self-management improves medication adherence and markers of asthma control. J Allergy Clin Immunol. 2009;123(Suppl 4):840-6.

5. Patton D, Woods S, Franklin D Jr, Cattie J, Heaton R, Collier A, Marra C, Clifford D, Gelman B, McArthur J, Morgello S, Simpson D, McCutchan A,
Grant I, Charter group. Relationship of medication management testrevised (MMT-R) performance to neuropsychological functioning and antiretroviral adherence in adults with HIV. AIDS Behav. 2012;16(Suppl 8):2286-96

6. Kripalani S, Henderson L, Chiu E, Robertson R, Kolm P, Jacobson T. Predictors of medication self-management skill in a low-literacy population. J Gen Intern Med. 2006;21(8):852-6.

7. Bailey S, Oramasionwu C, Wolf M. Rethinking adherence: a health literacy-informed model of medication self-management. J Health Commun. 2013;18(Suppl 1):20-30.

8. Gadoury M, Schwartzman K, Rouleau M, Maltais F, Julien M, Beaupre' A, Renzie PR, Be'gin R, Nault D, Bourbeau J, for the chronic obstructive pulmonary disease axis of the respiratory health network. Self-management reduces both short- and long-term hospitalisation in COPD. Eur Respir J. 2005;26(5):853-7.

9. Hutchison LC, Jones SK, West DS, Wei JY. Assessment of medication management by community living elderly persons with two standardized assessment tools; a cross-sectional study. Am J Geriatr Pharmacother 2006:4:144-53.

10. Abdullahi A, Hassan A, Kadarman N, Saleh A, Baraya S, Lua P. Food safety knowledge, attitude, and practice toward compliance with abattoir laws among the abattoir workers in Malaysia. J Gen Med. 2016:9:79-87.

11. Gupta U, Sharma S, Sheth PD, Jehva J, Chaudhary RR. Improving medicine usage through patient information leaflets in India. Trop Doct. 2005;35:164-6.

12. Wolf M, Davis T, Tilson H, Bass lii P, Parker R. Misunderstanding of prescription drug warning labels among patients with low literacy. Am Soc Health Syst Pharm. 2006;63:1048-55.

13. Teshome DF, Bekele KB, Habitu YA, Gelagay AA. Medication adherence and its associated factors among hypertensive patients attending the Debre Tabor General Hospital, northwest Ethiopia. Integr Blood Press Control. 2017;10:1-7.

14. Patel S, Dowse R. Understanding the medicines information-seeking behavior and information needs of South African long-term patients with limited literacy skills. Health Expect. 2013;18:1494-507.

15. Koh K, Brach C, Harris M, Parchman L. A proposed 'health literate care model' would constitute a systems approach to improving patients' engagement in care. Health Aff. 2013;32(Suppl 2):357-67.

16. Atak N, Gurkan T, Kose K. The effect of education on knowledge, self management behaviours and self efficacy of patients with type 2 diabetes. Aust J Adv Nurs. 2008;26(Suppl 2):66-74.

17. Johnson VR, Jacobson KL, Gazmararian JA, Blake SC. Does social support help limited-literacy patients with medication adherence? a mixed methods study of patients in the pharmacy intervention for limited literacy (PILL) study. Patient Educ Couns. 2010;79:14-24.

18. Ngoh LN. Health literacy: a barrier to pharmacist-patient communication and medication adherence. Pharm Today. 2009;15(Suppl 8):45-57.

19. Mbuagbaw L, Ndongmanji E. Patients' understanding of prescription instructions in a semi-urban setting in Cameroon. Patient Educ Couns. 2012;88:147-51.

20. Henrich N, Tsao N, Gastonguay L, Lynd L, Marra CA. BC medication management project: perspectives of pharmacists, patients and physicians. Can Pharm J (Ott). 2015;148:90-100.

21. Soller RW, Vogt E. Positioning patient counselling as a measurable cornerstone of medication therapy management. Int J Pharm Pract. 2010;18:249-51.

22. Riley K. Enhanced medication management services in the community: a win-win proposal from an economic, clinical and humanistic perspective. Can Pharm J. 2013;146:162-8.

23. Moczygemba LR, Cox LS, Marks SA, Robinson MA, Goode J-VR, Jafari N. Homeless patients perceptions about using cell phones to manage medications and attend appointments. Int J Pharm Pract. 2017:25:220-30.

24. Tsao NW, Khakban A, Gastonguay L, Zafari Z, Lynd LD, Marra CA. Opinions and preferences of British Columbia pharmacists and physicians on medication management services. Can Pharm J (Ott). 2017;150:42-51.

\section{Publisher's Note}

Springer Nature remains neutral with regard to jurisdictional claims in published maps and institutional affiliations. 